\title{
Features of the honey bee APIS mellifera genome versus fruit fly drosophila melanogaster
}

\begin{abstract}
The analysis of the nuclear and the mitochondrial genomes of the honey bee Apis mellifera in comparison with the well-annotated, finished fruit fly Drosophila melanogastergenome was presented in this article. The nuclear genome of the honey bee has about 245 millions b.p. which distributed in 16 chromosomes and contains about 10 thousands genes. The mitochondrial genome of the A.mellifera has about 16 thousands b.p. which located in mitochondrion's and contains 35 genes. The nuclear genome of the fruit fly has about 144millions b.p. which distributed in 4 chromosomes and contains about 17 thousands genes. The mitochondrial genome of the D. melanogaster has about 19 thousands b.p. which located in mitochondrion's and contains 37 genes. Despite the full sequencing of the nuclear and the mitochondrial genomes of the A. melliferathe function of several genes and loci of A. mellifera are not disclosed fully. A comparative analysis of the genomes of A. Mellifera and D. melanogaster using bioinformatics techniques allowed to reveal the features of the structure and function of the honey bee A. mellifera genome. The genome of $A$. Mellifera have more similarity with the vertebrate genome than D. melanogaster. The genome of $A$. Mellifera contains less genes of the native immunity, of detoxification enzymes, of cuticle proteins and taste receptors compared with D. melanogaster. However, A. mellifera contains new genes associated with olfactory receptors, the processing of pollen and nectar, poison organs, wax glands, caste determination and labor division which absent at D. melanogaster. Probably, this is due to the ecology of bees and their social evolution.
\end{abstract}

Keywords: nuclear genome, mitochondrial genome, apis mellifera, honey bee, genes, chromosomes
Volume 3 Issue 2 - 2016

\section{Ilyasov RA}

Institute of Biochemistry and Genetics, Russian Academy of Sciences, Russia

\begin{abstract}
Correspondence: llyasov RA, Institute of Biochemistry and Genetics, Ufa Research Center, Russian Academy of Sciences, Ufa, 450054, Russia, Email apismell@hotmail.com
\end{abstract}

Received: August 25, 2016 | Published: September 19, 2016
Abbreviations: RTK, receptor tyrosine kinase; HH, hedgehog; TGF-B, transforming growth factor-B; JAK, janus kinase; STAT, signal transducer and activator of transcription; EAAT, excitatory amino acid transporters; SCR, sex combs reduced; ANTP, antennapedia, ABD-A, abdominal-A; EN, engrailed; MSH, muscle segment homeobox

\section{Introduction}

There was only two sequenced genomes of the two Dipterans species Drosophila melanogaster ${ }^{1}$ and Anophelesgambiae ${ }^{2}$ before the recent sequencing of the honey bee Apis mellifera(Hymenoptera), Tribolium castaneum and Bombyx mori genomes. Hymenoptera diverged from Dipterans about 300million years ago, and recent phylogenetic evidence implies that the Apis are the most distant group of holometabolous insects from Drosophila. ${ }^{3-5}$ The honeybee genome was compared with the well-annotated, finished $D$. melanogaster genome. The D.melanogaster genome is most studied of all the genomes. Despite $A$. mellifera is very economically important insect a few studies of it full genome have been published. Therefore, comparative analysis of two genomes the A. mellifera and $D$. melanogaster is very interesting. Differences between A. mellifera and $D$. melanogaster caused by not only the nucleotide polymorphism of the genes but also by their different epigenetical regulation. Superficially, A. mellifera development is similar to that of $D$. melanogaster, in that it is a holometabolous. However, A. mellifera are different in their development and biology from the D. melanogaster in a number of ways. There is a hypothesis that all the differences that are observed between A. mellifera and D. melanogaster, have occurred since their divergence. This hypothesis is confirmed by the differences observed between A. mellifera and D. melanogaster in the early stages of development. ${ }^{6-8}$ Thus, A. mellifera use haplodiploidy to determine sex, a process different from that of sex determination in D. melanogaster. The adult honey bee's A.mellifera has several novel evolutionary innovations not present in D. melanogaster, including poison organs and wax glands. Most important are the caste determination and labor division associated with the social nature of the honey bee. ${ }^{5}$

The nuclear genome of the honey bees A. mellifera has 246927000 b.p. which subdivided into 16 chromosomes and containing 10157 genes(Gene Bank access AADG00000000). ${ }^{6,7}$ The mitochondrial genome of the honey bees has 16343 b.p. which represented by a circular molecule of DNA and containing 35 genes(Gene Bank access NC 001566). ${ }^{8}$ All chromosomes of the honey bees has different sizes: LG 1(NC_007070) 30000 b.p. contains 1669 genes(25 non coding genes); LG 2(NC_007071) 15500 b.p. - 814 genes(27 non coding genes); LG 3( NC_007072 ) 13200 b.p. - 735 genes(20 non coding genes); LG 4( NC_007073 ) 12700 b.p. - 709 genes(46 non coding genes); LG 5(NC 007074) 14400 b.p. - 874 genes(13 non coding genes); LG 6(NC_007075) 18500 b. p. - 844 genes(15 non coding genes); LG 7(NC_007076) 13200 b.p. - 596 genes(9 non coding genes); LG 8( NC_007077 ) 13500 b.p. - 873 genes(33 non coding genes); LG 9( NC_007078 ) 11100 b.p. - 584 genes(17 non coding genes); LG 10( NC__007079) 13000 b.p. - 768 genes(11 non coding genes); LG 11(NC_007080) 14700 b.p. - 968 genes(16 non coding genes); LG 12( NC_007081 ) 11900 b.p. - 504 genes(14 non coding genes); LG 13( NC_007082 ) 10300 b.p. - 418 genes(13 non coding genes); LG 14( NC_007083 ) 10300 b.p. - 612 genes(8 non coding genes); LG 15( NC_007084 ) 10200 b.p. - 730 genes(30 non coding genes); LG 16( NC_007085) 7200 b.p. - 420 genes(26 non coding 
genes)(Gene Bank - http://www.ncbi.nlm.nih.gov, EnsemblMetazoa - http://metazoa.ensembl.org). For comparison, the nuclear genome of the fruit flies D. melanogaster has 143726000 which sub divided into four chromosomes(2 large, 1 small autosomes and the $\mathrm{X} / \mathrm{Y}$ sex chromosomes) and containing 17651 genes(3 384 non coding genes)(Gene Bank access GCA_000001215.4). ${ }^{1}$ The mitochondrial genome of the fruit flies has 19524 b.p. which represented by a circular molecule of DNA and containing 37 genes(Gene Bank access NC_024511.2). ${ }^{5}$ All chromosomes of the fruit flies has different sizes: 2L( NT_033779.5) 23510 b.p. contains 3485 genes(770 non coding genes); 3L(NT_037436.4) 28110 b.p. contains 3453 genes(666 non coding genes); 4( NC_004353.4) 1350 b.p. contains 112 genes(26 non coding genes); $\mathrm{X}\left(\mathrm{NC} \_004354.4\right) 23540$ b.p. contains 2661 genes(408 non coding genes); Y( NC 024512.1) 3670 b.p. contains 113 genes(28 non coding genes)(Gene Bank- http://www.ncbi.nlm. nih.gov, Ensembl Metazoa - http://metazoa.ensembl.org).

The nuclear and mitochondrial genome of $A$. mellifera differ from D. Melanogaster by high containing of AT-rich regions. Since the A. mellifera's nuclear genome contains $67 \%$ and the mitochondrial genome - $85 \%$ AT whereas $D$. melanogaster's nuclear genome contains $58 \%$ and the mitochondrial genome - 79\% AT nucleotides. ${ }^{9}$ The nuclear and mitochondrial genome of A. mellifera characterized by greater spatial heterogeneity of AT-rich areas, higher content of CpG islands and absence of the most common families of transposones than at $D$. melanogaster. The genes of $A$. mellifera predominantly located in AT-rich areas and characterized by high content of GC nucleotides. The A and T nucleotides of AT-rich areas in protein coding genes of A. mellifera are located in second and third positions predominantly. ${ }^{6,7}$ The structure and localization of most common genes in A. mellifera differ from $D$. melanogaster. In the A. mellifera mitochondrial genome 11 genes of tRNA has shift position as compared with D. melanogaster. The genetic code of A. mellifera similar to $D$. Melanogaster but two anticodons of tRNA differ(tRNALYS -TTT, tRNASER - TCT in A. mellifera andtRNALYS - CTT, tRNASER GCT in D. melanogaster). ${ }^{8}$

Some nuclear genes of the A. mellifera are orthologs to the $D$. melanogaster genes, which has differences in sizes. Thus in $A$. mellifera the genes of Yellow/Major Royal Jelly Protein is larger, the genes of cuticular proteins is smaller, the genes of odorant receptors is larger, the genes of gustatory receptors is smaller, the genes of immunity is smaller, the detoxification genes is smaller than in $D$. Melanogaste. ${ }^{6,10}$ In the $A$. mellifera genes the trans versions occurred more frequently than transition whereas in D.melanogaster it is conversely. In the A. mellifera genes trans versions occurred on third position of codons. Some genes of $A$. mellifera arisen as a result of evolutionary changes of the genes of common with D.melanogaster ancestors. Thus, the gene of $A$. mellifera encoding the major protein of royal jelly is derived from the ancient gene yellow, which presented in D. melanogaster. Many genes of A. mellifera and D.melanogasteris similar, butsome genes of D. Melanogaster is absent in A. mellifera. For example, in A.mellifera, the genes of WNT cell signalling pathways as Hedgehog(HH), Transforming Growth Factor-B(TGF-B), Receptor Tyrosine Kinase(RTK), NOTCH, Janus Kinase(JAK), Signal Transducer and Activator of Transcription(STAT) are similar with D. melanogaster. However, the genes of cell signalling systems(Terminal Embryo Fate, TRUNK, TORSO), of component of the dorso-ventral signalling system(GURKEN), of the G-proteincoupled receptor(mGluR-like) family(BOSS) are missing from the $A$. mellifera genome. ${ }^{6}$

Some genes of the D. melanogaster has novel features in the
A. mellifera. Thus, the gene of the Glucose-methanol-choline oxidoreductases family(NINAG) in A. mellifera presents as two putative NINAG-like genes, the gene of the receptor protein tyrosine kinase family(INR) in A. mellifera is duplicated; the gene of the phosphor lipase $\mathrm{C}$ family(NORPA) in A. mellifera is duplicated, the gene of the photoreceptor-cell-specific nuclear receptor family $(P N R)$ in $A$. mellifera presents as three genes versus two genes in D.Melanogaster, the geneof the TRPA subfamily of transient receptor potential channels family(TRPA1) are missing in D. melanogaster, but has two extra TRPA channels(GB14005 and GB16385) in $A$. mellifera; the gene of the ligand-gated ion channels family(NACR) in A. mellifera presents as 11 subunits instead of 10 in D. Melanogaster, the geneof the ligand-gated ion channels family(NMDAR) in $A$. mellifera presents as 3 genes instead of 2 in D. Melanogaster, the geneof the excitatory amino acid transporters family(EAAT) in $A$. mellifera presents as 5 genes instead of 2 in D. Melanogaster. ${ }^{6}$ In A. mellifera 96 homeobox domains were found in 74 genes, similar to D. melanogaster. More than $90 \%$ identity represented by homeobox genes(Sex Combs Reduced(SCR), Antennapedia(ANTP), AbdominalA(ABD-A); Engrailed(EN), Muscle Segment Homeobox(MSH)). For the remaining $A$. mellifera genes, a D.melanogaster homologue is not known. This indicates that structurally homologous genes are involved in the control of A. mellifera and D. Melanogasterdevelopment. ${ }^{6,11}$

The nuclear genes of A. mellifera which responsible for circadian rhythms(CRY-M, CLK, CYC, PDP1, VRI, PER), RNA interference(RNAi) and DNA methylation(381 genes in eggs and sperm of A. mellifera with $\mathrm{CpG}$ methylation) have more similarity with genes of vertebrate than with genes of $D$. Melanogaste. ${ }^{12-15}$ The circadian rhythms genes Timeless(TIM1) and Crypto chrome(DCRY) of $D$. melanogaster are absent in A. mellifera genome. The similarity with vertebrate may be explained by the parallel evolution of the some genes during adaptation to the environment conditions. The genome of $A$. mellifera contains less genes of the native immunity, of detoxification enzymes, of cuticle proteins and taste receptors compared with $D$. melanogaster. However, A. mellifera contains new genes associated with olfactory receptors, the processing of pollen and nectar which absent at $D$. melanogaster. Probably, this is due to the ecology of bees and their social organization., ${ }^{5,16}$

The rate of the evolutionary transformations of the nuclear and mitochondrial genome of $A$. mellifera less than in D. melanogaster. However, the genome of $A$. mellifera diverged more from common ancestor than D. Melanogaster. ${ }^{17,18}$ Probably, this is due to the small effective population size of $A$. mellifera and to low rate of the reverse mutation compared with D. Melanogaster. ${ }^{19}$ Micro RNA(miRNA) of the nuclear genome of A. mellifera plays an important role in the regulation of social organization and caste differentiation via posttranscriptional regulation of gene expression. About 300 honey bee miRNAs deposited in miRBase (http://www.mirbase.org). ${ }^{20}$ For example, differentially expressed miRNAs between 4day-old queen and worker larvae of honey bees: up-regulated in queen larvae(amebantam, ame-let-7, ame-mir-10, ame-mir-100, ame-mir-6001-3p); equally expressed in queen larvae(ame-mir-11, ame-mir-1175, amemir-190, ame-mir-6065, ame-mir-989); down-regulated in queen larvae(ame-mir-13b, ame-mir-252a, ame-mir-2765-5p, ame-mir-996, ame-mir-9a) ${ }^{21}$ In the nuclear genome of $A$. mellifera found miRNA, which characterized by caste specific expression: the miRNA C5599F most expressed in the queens, C689F - in the pupaes, C5560 - in the pupaes of workers. ${ }^{7}$ Thus, the genome of $A$. mellifera have more similarity with the vertebrate genome than $D$. melanogaster. The genome of $A$. mellifera contains less genes of the native immunity, 
of detoxification enzymes, of cuticle proteins and taste receptors compared with $D$. melanogaster. However, A. mellifera contains new genes associated with olfactory receptors, the processing of pollen and nectar, poison organs, wax glands, caste determination and labor division which absent at D. melanogaster. Probably, this is due to the ecology of bees and their social evolution. A comparative analysis of the genomes of $A$. mellifera and $D$. melanogaster using bioinformatics techniques allowed revealing the features of the structure and function of the honey bee A. mellifera genome. It is very important for understanding the human genome also.

\section{Acknowledgments}

This research was supported by the Russian Foundation for Basic Research(grant No. 14-04-97084 r povolzhye a) and was carried out on equipment available at the Biomica Center for Collective Use of the Division of Biochemical Methods of Analysis and Nano biotechnology of the Agidel Resource Center for the Collective Using.

\section{Conflict of interest}

Author declares that there is no conflict of interest.

\section{References}

1. Adams MD, Celniker SE, Holt RA, et al. The genome sequence of Drosophila melanogaster. Science. 2000;287(5461):2185-2195.

2. Holt RA, Subramanian GM, Halpern A, et al. The genome sequence of the malaria mosquito Anopheles gambiae. Science. 2002;298(5591):129-149.

3. Whiting MF. Phylogeny of the holometabolous insect orders: Molecular evidence. Zoologica Scripta. 2002;31(1):3-15.

4. Krauss V, Pecyna M, Kurz K, et al. Phylogenetic mapping of intron positions: A case study of translation initiation factor eIF $2 \gamma$. Mol Biol Evol. 2005;22(1):74-84.

5. Dearden PK, Wilson MJ, Sablan L, et al. Patterns of conservation and change in honey bee developmental genes. Genome Research. 2006;16(11):1376-1384.

6. Weinstock GM, Robinson GE, Gibbs RA, et al. Insights into socia insects from the genome of the honeybee Apis mellifera. Nature. 2006;443(7114):931-949.

7. Whitfield CW, Behura SK, Berlocher SH, et al. Thrice out of Africa: ancient and recent expansions of the honey bee, Apis mellifera. Science. 2006;314(5799):642-645.
8. Crozier RH, Crozier YC. The mitochondrial genome of the honeybee Apis mellifera: complete sequence and genome organization. Genetics. 1993;113(1):97-117.

9. Jukes TH, Bhushan V. Silent nucleotide substitutions and $\mathrm{G}+\mathrm{C}$ content of some mitochondrial and bacterial genes. $J \mathrm{Mol}$ Evol. 1986;24(1-2):39-44.

10. Wang Y, Odemer R, Rosenkranz P, et al. Putative orthologues of genetically identified Drosophila melanogaster chitin producing and organising genes in Apis mellifera. Apidologie. 2014;45(6):733-747.

11. Walldorf U, Fleig R, Gehring WJ. Comparison of homeobox-containing genes of the honeybee and Drosophila. Proc Natl Acad Sci U S A. 1989;86(24):9971-9975.

12. Toma DP, Bloch G, Moore D, et al. Changes in period mRNA levels in the brain and division of labor in honey bee colonies. Proc Natl Acad Sci USA. 2000;97(12):6914-6919.

13. Rubin EB, Shemesh Y, Cohen M, et al. Molecular and phylogenetic analyses reveal mammalian-like clockwork in the honey bee (Apis mellifera) and shed new light on the molecular evolution of the circadian clock. Genome Res. 2006;16(11):1352-1365.

14. Elango N, Hunt BG, Goodisman MAD, et al. DNA methylation is widespread and associated with differential gene expression in castes of the honeybee, Apis mellifera. Proc Natl Acad Sci USA. 2009;106(27):11206-11211.

15. Drewell RA, Bush EC, Remnant EJ, et al. The dynamic DNA methylation cycle from egg to sperm in the honey bee Apis mellifera. Development. 2014;141(13):2702-2711.

16. Wallberg A, Han F, Wellhagen G, et al. A worldwide survey of genome sequence variation provides insight into the evolutionary history of the honeybee Apis mellifera. Nat Genet. 2014;46(10):1081-1088.

17. Crozier RH, Crozier YC, Mackinlay AG. The CO-I and CO-II region of the honeybee mitochondrial DNA: evidence for variation in insect mitochondrial evolutionary rates. Mol Biol Evol. 1989;6(4):399-695.

18. Crozier RH, Crozier YC. The cytochrome b and ATPase genes of honeybee mitochondrial DNA. Mol Biol Evol. 1992;9(3):474-482.

19. Crozier RH. Genetical structure of social insect populations. In: Markl $\mathrm{H}$, editor. Evolution of Social Behavior: Hypotheses and Empirical Tests. Dahlem konfer enzem, Weinheim: Verlag Chemie; 1980. p. 129-146.

20. Ashby R, Forêt S, Searle I, et al. MicroRNAs in honey bee caste determination. Scientific Reports. 2016;6:18794.

21. Shi YY, Zheng HJ, Pan QZ, et al. Differentially expressed microRNAs between queen and worker larvae of the honey bee (Apis mellifera). Apidologie. 2015;46(1):35-45. 